\title{
Global Neuroinformatics: The International Neuroinformatics Coordinating Facility
}

\author{
Jan G. Bjaalie ${ }^{1}$ and Sten Grillner ${ }^{1,2}$ \\ ${ }^{1}$ International Neuroinformatics Coordinating Facility and ${ }^{2}$ Nobel Institute for Neurophysiology, Department of Neuroscience, Karolinska Institutet, \\ SE-171 77 Stockholm, Sweden
}

There is a growing awareness in the neuroscience community of the need for databases extending from genes to cognition and disease mechanisms. Such databases are important for data sharing as well as for modeling and use of computational tools at different levels. The development of this area, neuroinformatics, would ben-

Received Feb. 8, 2007; revised Feb. 20, 2007; accepted Feb. 20, 2007.

J.G.B. is the Executive Director of the INCF. S.G. is the Chair of the Governing Board of the INCF. The members of the INCF Governing Board contribute to the development of the INCF, together with the INCF national nodes. Previous working groups of the Organization for Economic Cooperation and Development (OECD) Global Science Forum (GSF) have prepared the background analysis that resulted in the recommendation of the OECD Ministers of Science to establish the INCF. Chairs and cochairs of these working groups were Stephen H. Koslow (United States), Shun-ichi Amari (Japan), and Sten Grillner (Europe). The operations of the INCF build on the Understanding and Business Plan of the INCF. This article describes principles only. INCF strategies and actions are subject to continuous monitoring and approval by the INCF Governing Board. Role of the OECD: OECD is an intergovernmental organization which provides a forum for analysis, development, and reform of economic and social policies. The division that represents the basis for the establishment of the INCF is the Directorate for Science, Technology, and Industry. This Directorate provides policy advice in areas such as biotechnology, telecommunications, and information services. It includes the GSF as a venue for meetings of senior science policy officials of $O E C D$ countries with the goal of identifying and maximizing opportunities for international cooperation. GSF special-purpose working groups perform technical analyses and develop findings and recommendations for actions by governments. The first such working group in the area of neuroinformatics was established in 1996, under the forerunner of GSF, the MegaScience Forum, and delivered a report in 1999 (http://www.inf.org/ med/Report_OECD_MSF_1999.pdf). At the request of the GSF, a further examination was performed, leading to a second report in 2002 (http:// www.incf.org/med/Report_OECD_GSF_2002.pdf). The latter report was endorsed at the OECD Science and Technology Ministerial meeting in January 2004. Sixteen countries, as well as the European Commission, then elaborated the legal basis and platform for the INCF.

Correspondence should be addressed to Jan G. Bjaalie, INCF Secretariat, Karolinska Institutet, Nobels väg 15 A, SE-171 77 Stockholm, Sweden. Email:jan.bjaalie@incf.se.

DOI:10.1523/JNEUROSCI.0558-07.2007

Copyright $\odot$ 2007 Society for Neuroscience $\quad$ 0270-6474/07/273613-03\$15.00/0 efit from a major concerted action. For this reason 12 member countries of the Organization for Economic Cooperation and Development have formed the International Neuroinformatics Coordinating Facility (INCF), an organization that will facilitate the development of neuroinformatics (http://www.incf.org). This effort is financed by contributions from each member nation (Belgium, Czech Republic, Finland, France, Germany, Italy, Japan, The Netherlands, Norway, Sweden, Switzerland, and the United States), as well as from the European Commission. The central Secretariat of the INCF in Stockholm will have a staff of $\sim 10$, including the Executive Director, Program Managers, and other staff. We outline here the objectives of the INCF and the resources that are being developed for neuroscience.

The mission of the INCF is to (1) coordinate and foster international activities in neuroinformatics; (2) contribute to the development of scalable, portable, and extensible applications that can be used for furthering our knowledge of the human brain and its diseases; (3) contribute to the development and maintenance of specific database and other computational infrastructures and support mechanisms; and (4) focus on developing mechanisms for the seamless flow of information and knowledge between academia, private enterprises, and the publication industry.

An extensive analysis and a series of platform documents serve as a basis for the operations that are to be performed by the INCF (Table 1). Neuroinformatics, as defined in the context of the INCF, is an interdisciplinary research area combining neuroscience with information sciencel technology. Neuroinformatics deals with the development of neuroscience data and knowledge bases together with computational models and analytical tools for the sharing, integration, and analysis of experimental data, discovery research, and the advancement of theories of nervous system function (Fig. 1). The ultimate goal of the INCF is to accelerate progress in the understanding of brain function. It also may serve as a source of inspiration for information sciences and development of technologies using principles of neural processing in the brain. Understanding the brain requires integration of heterogeneous and complex data collected at multiple levels of investigation.

\section{Principal Work Program}

The principal work program of the INCF covers large areas of neuroinformatics, as listed in Table 2. Main deliverables include (1) web-based portal services, focused on access to interoperable and integrated databases and tools covering selected areas, (2) new approaches to use of standardized terminologies and standards for data production, and (3) novel training opportunities. Some aspects of these areas, which will be of direct relevance for neuroscientists, are outlined below.

Portals to neuroinformatics resources A large number of neuroinformatics resources, ranging from data repositories to 
advanced database applications and including shared tools and computational models, are currently available. A listing of many of the available resources can be found at the Neuroscience Database Gateway, hosted by The Society for Neuroscience (http://www.sfn.org/ndg). Another important and supplementary initiative is the Neuroscience Information Framework (http://neurogateway.org). The INCF will assist the community with the creation of an inventory of available resources, with the starting point being the activities and priorities of the INCF national nodes in each of the member countries. Furthermore, the INCF will focus on the development of portals providing access to different research areas and domains, primarily to areas that are already represented by a critical mass of distributed neuroinformatics resources. The motivation is not only to assist neuroscientists in finding individual resources but to facilitate rapid and smart access to relevant combinations of resources.

Such integration of existing resources is demanding. It serves two primary purposes. The first purpose is obviously to extract the most out of the existing resources and to make the deliverables of already funded research and development as accessible and useful to the neuroscience community as possible. The second purpose is to prepare the bases for future projects, in charting the present bottlenecks for integration, within the scientific or technical domain or at the sociological level. After the first analysis of such systems integrations, and drawing on the initial experiences, the INCF will map the road ahead in terms of services that will be developed within the scope of the INCF work plan. Interaction with industry in terms of developing products is not a main focus of the INCF but will be taken into consideration (e.g., to the extent that sustainability of resources will benefit from introducing a commercial component). The latter is also relevant to one goal of the INCF, the development of capabilities for transfer of knowledge and technology.

\section{Standards and guidelines}

Neuroscience produces heterogeneous datasets. The field is divided into many disciplines with different practices, and different investigators often use different terminologies. Producing data and metadata suitable for sharing through interoperable and federated database applications prepared for smart searches and analyses may therefore appear compli-

\section{Table 1. Platform documents for the INCF}

\begin{tabular}{lc}
\hline Document & Description \\
\hline $\begin{array}{l}\text { Understanding for the International Neuroinformatics Coordinating } \\
\text { Facility (http://www.incf.org/med/INCF_Understanding.pdf) }\end{array}$ & $\begin{array}{c}\text { The legal document signed by all participants in the } \\
\text { INCF that sets the framework for joining the INCF }\end{array}$ \\
$\begin{array}{l}\text { Business Plan for the International Neuroinformatics Coordinating } \\
\text { Facility (http://www.incf.org/med/INCF_BusinessPlan.pdf) }\end{array}$ & $\begin{array}{c}\text { The document that describes the mission and operating } \\
\text { procedure of the INCF } \\
\text { Program in International Neuroinformatics (http://www.incf.org/ } \\
\text { The framework document for a future funding scheme } \\
\text { for international neuroinformatics }\end{array}$ \\
\hline
\end{tabular}

\section{Neuroinformatics}

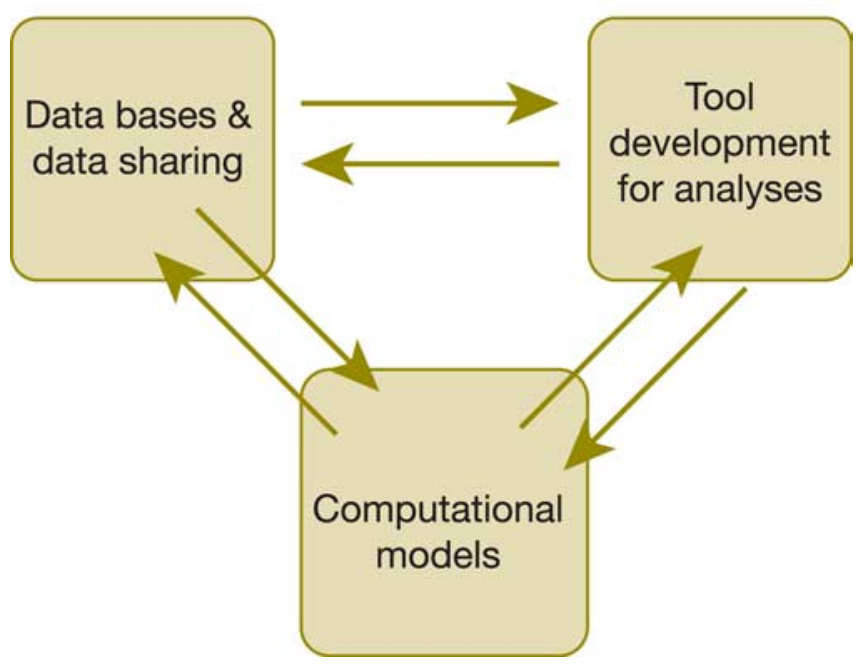

Figure 1. The three foci of neuroinformatics.

cated. With new technological developments and possibilities emerging in data production and data management, it appears logical and necessary to gradually develop standards at all levels of research. Standardized terminologies and standards for data production will make data less context dependent and will provide new opportunities for comparing and integrating data from different laboratories. In developing standards, it is useful to distinguish between exploratory and production-oriented activity. Much of neuroscience involves methods that are intricate and difficult to fully standardize. Research itself may even lead to modification of standards and terminology. However, large laboratories, or consortia of laboratories, may operate productionoriented facilities using highly standardized methods, including high throughput technologies. The INCF will interact with such production-oriented facilities to optimize communication among the facilities and between the facilities and the larger research community. The primary goal will be to contribute to the development and use of standards and guidelines
Table 2. Principal work program for the INCF

Databases that cover basic and clinical neuroscience from the level of the gene to behavior

Infrastructure, such as portals, communication channels, database federations, and grid middleware

Tools, such as simulation environments for computational neuroscience, computational devices, data analysis, data mining, data warehousing, and middleware

Construction and dissemination of models of the nervous system and simulation of neural processes Guidelines, standards, and ontologies Interdisciplinary training programs

Consensus on strategies and priorities and joint, possibly novel, funding agreements to carry out specific projects or meet specific goals

General transfer of knowledge and technology

The INCF will facilitate and contribute to the development and access to and/or dissemination of capabilities as listed.

for as many domains of neuroscience as practical. The INCF will promote the establishment of interoperable database resources and facilitate the development of standards and guidelines for data production, analyses, and use of terminologies.

\section{Interdisciplinary training}

Neuroinformatics comprises many disciplines, and development of neuroinfor- 

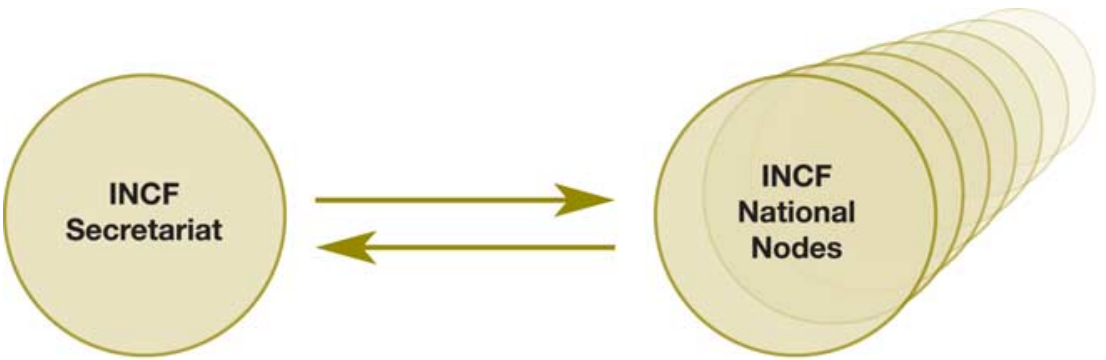

- Coordination of tasks

- Analyses and recommendations

- Specialized developments

- National infrastructure

- Infrastructure developments

- International, interdisciplinary training

- Standards, guidelines, and ontologies

- Portal to resources

Figure 2. Overview of tasks of the Secretariat and national nodes. Decisions on the goals and actions of the Secretariat are made by the Governing Board, with representatives from the member countries.

matics applications, whether databases, tools, or simulation environments, requires interaction between developers and end users. The developers may not have experience in neuroscience, and similarly, the end users may not have sufficient experience in information sciences and related disciplines. Interdisciplinary training will have to assist those with a background in computational methods to get a working knowledge of experimental brain sciences at some level and to help the life scientists to become adept with requirements imposed by computational principles and methods. Crossdisciplinary training is challenging but fundamental to the success of neuroinformatics and neuroscience in general. To raise the skills of the community of neuroinformatics researchers, several tasks must be addressed. First, there is a need to develop a systematic overview of training needs. The results of the INCF workshop series (see below) will provide important input in this respect. Second, good practice schemes for training are needed, tak- ing into account the requirements of the research community and advances in neuroscience and neuroscience-related technologies. Third, exchange schemes for early-career scientists need to be facilitated, offering opportunities for specialized practitioners to be exposed to selected areas and fields that will be of importance for their work.

\section{Work Model of the INCF}

A key element in the INCF operation is to work closely with the scientific community in adjusting and translating the work plan to practical action. To this end, INCF will organize a series of topical workshops that will cover not only technical issues of primary importance for neuroinformatics, but also research topics coupled to databasing of neuroscience data, tool developments, or modeling of nervous system functions. Each workshop has 10-15 invited participants, selected on the basis of their expert skills in the particular area of the workshop. To date, workshops on Large-Scale Modeling of the Nervous Sys- tem and Mouse and Rat Brain Digital Atlasing Systems have been organized. Reports with recommendations for action are in preparation and will be made available via the INCF website. Upcoming topics to be covered by the workshop series include Neuroanatomical Nomenclature and Taxonomy, Sustainability for Neuroscience Databases, and Portals to Neuroinformatics Resources. Follow-up from the workshops will be taken care of by establishing expert panels for the different areas covered by the workshops and by connecting the expert panels closely to staff in the INCF Secretariat and in national nodes (Fig. 2). To the extent possible, actions of the INCF will always seek to build on a careful mapping of existing resources and, when possible, to prepare for the full use of these in combination with new developments.

\section{Future Perspectives in Neuroinformatics}

The short-term priorities of the INCF are to create inventories of neuroinformatics resources available in the member nations and to launch portals to topical, limited research areas as well as to analytical tools. In a longer time perspective, the INCF will prioritize actions that will lead to an improved environment for data sharing, to the development of larger, interoperable databases in selected areas, and to easier access to standardized, shared tools, as well as to shared environments for simulations and computational analysis. The INCF effort will progress by focusing on selected areas and building on success stories and experiences. It should become a significant achievement with neuroinformatics playing the same role in the development of basic and clinical neuroscience as bioinformatics has for genomics and proteomics. 